\title{
Synthetic emmprin peptides with chitobiose substitution stimulate MMP-2 production by fibroblasts
}

Takehito Kawakami ${ }^{1,2}$, Tetsuro Sameshima ${ }^{3}$, Hironobu Hojo ${ }^{4}$, Kaori Koga ${ }^{1}$, Yoshiaki Nakahara ${ }^{4}$, Bryan P Toole ${ }^{5}$, Junji Suzumiya ${ }^{6}$, Yasunori Okada ${ }^{7}$, Akinori Iwasaki $^{2}$ and Kazuki Nabeshima ${ }^{*}$

\begin{abstract}
Background: Emmprin, a glycoprotein containing two Ig domains, is enriched on tumor cell surfaces and stimulates matrix metalloproteinase (MMP) production by adjacent stromal cells. Its first Ig domain (ECI) contains the biologically active site. The dependence of emmprin activity on N-glycosylation is controversial. We investigated whether synthetic $\mathrm{ECl}$ with the shortest sugar is functionally active.
\end{abstract}

Methods: The whole ECI peptides carrying sugar chains, a chitobiose unit or N-linked core pentasaccharide, were synthesized by the thioester method and added to fibroblasts to examine whether they stimulate MMP-2 production.

Results: $\mathrm{ECl}$ carrying a chitobiose unit, $\mathrm{ECl}(\mathrm{GlCNAC})_{2}$, but not $\mathrm{ECl}$ without a chitobiose unit or the chitobiose unit alone, dose-dependently stimulated MMP-2 production by fibroblasts. $\mathrm{ECl}$ with longer chitobiose units, $\mathrm{ECl}-\left[(\mathrm{Man})_{3}\right.$ $\left.(\mathrm{GlcNAc})_{2}\right]$, also stimulated MMP-2 production, but the extent of its stimulation was lower than that of $\mathrm{ECl}-(\mathrm{GlcNAc})_{2}$.

Conclusions: Our results indicate that $\mathrm{ECl}$ can mimic emmprin activity when substituted with chitobiose, the disaccharide with which $\mathrm{N}$-glycosylation starts.

\section{Background}

Matrix metalloproteinases (MMPs) play an essential role in remodeling of extracellular matrices (ECMs) involved in various biological processes, such as inflammation, tissue regeneration and tumor invasion. Among the MMPs, gelatinase A (MMP-2) is the most abundant MMP and frequently correlates with malignant progression and invasive behavior of tumor cells [1]. In situ hybridization studies of human surgical specimens have shown that stromal fibroblasts are the predominant source of MMP2 in the majority of carcinomas [2-4]. Malignant cells stimulate nearby fibroblasts to produce MMPs via soluble cytokines and growth factors or through cell surface interactions mediated by plasma membrane proteins, such as emmprin [5]. Emmprin, also known as basigin/ $\mathrm{CD} 147$, is an integral plasma membrane glycoprotein of

\footnotetext{
* Correspondence: kaznabes@fukuoka-u.ac.jp

'Department of Pathology, Fukuoka University School of Medicine, 7-45-1

Nanakuma, Jonan-ku, Fukuoka 814-0180, Japan

Full list of author information is available at the end of the article
}

the Ig superfamily that contains two extracellular Ig domains [6]. Expression of emmprin is upregulated in human malignant tumors, such as breast, lung and bladder carcinomas, malignant melanomas, gliomas and lymphomas, compared with their normal counterparts [7-11]. In malignant tumors, emmprin acts as a modulator of tumor-stroma cross-talk, since it mediates not only MMP production but also tumor angiogenesis through the stimulation of vascular endothelial growth factor (VEGF) expression [12], induction of activated stromal myofibroblasts [13], and anchorage-independent growth and multidrug resistance in a hyaluronan-dependent fashion [14-16]. The activity-blocking monoclonal antibody (mAb), E11F4, recognizes the first Ig domain (ECI) of emmprin, implying that this region of emmprin contains the structure responsible for the activity of this protein $[5,6]$. Moreover, it is reported that emmprin-induced stimulation of MMP production in fibroblasts is dependent on $\mathrm{N}$-glycosylation of its extracellular domains $[17,18]$. However, it has been reported recently that

\section{Biomed Central}


nonglycosylated recombinant emmprin could stimulate fibroblasts to express the mRNAs of MMP-1, 2 and 3 [19]. The objective of the present study was to determine whether the activity of synthetic emmprin ECI peptides, with or without a chitobiose unit (GlcNAc-GlcNAc), the disaccharide with which $\mathrm{N}$-glycosylation starts, mimics that of emmprin. The results showed that synthetic ECI substituted with a chitobiose unit, but not ECI alone, stimulates fibroblasts to produce MMP-2.

\section{Methods}

\section{Peptides}

ECI (34-94th a.a.) carrying a chitobiose unit or N-linked core pentasaccharide at $\mathrm{Asn}^{44}$ was synthesized by the thioester method as described previously (Figure 1) [20-22]. ECI carrying one or two GlcNAc or N-linked core pentasaccharide $\left[(\mathrm{Man})_{3}(\mathrm{GlcNAc})_{2}\right]$ was dissolved in dimethyl sulfoxide (DMSO). The final concentration of DMSO in the culture medium was less than $0.01 \%$. At this concentration, the solvents alone showed no cytotoxic effects or any detectable effects on MMP production.

\section{Cell culture}

Human adult fibroblasts, MBT-3 and ST353, were obtained from a metastatic tumor in the cerebellum from a primary lung adenocarcinoma [23] and nonlesional dermis around nodular fasciitis [24], respectively. The tumor cell line FU-EPS-1 was established from a patient with epithelioid sarcoma who had not received any chemotherapy before surgical resection [25]. These cell lines were cultured in Dulbecco's modified Eagle's medium (DMEM) supplemented with $10 \%$ fetal bovine serum (FBS) and antibiotics $(100 \mathrm{U} / \mathrm{ml}$ penicillin $\mathrm{G}$ and $100 \mu \mathrm{g} / \mathrm{ml}$ streptomycin) in a humidified atmosphere of $5 \% \mathrm{CO}_{2}$ at $37^{\circ} \mathrm{C}$.

\section{Co-culture experiments}

Co-culture experiments were performed as described previously [24]. Briefly, cultures containing either fibroblasts, tumor cells or both were established in $20-\mathrm{mm}$

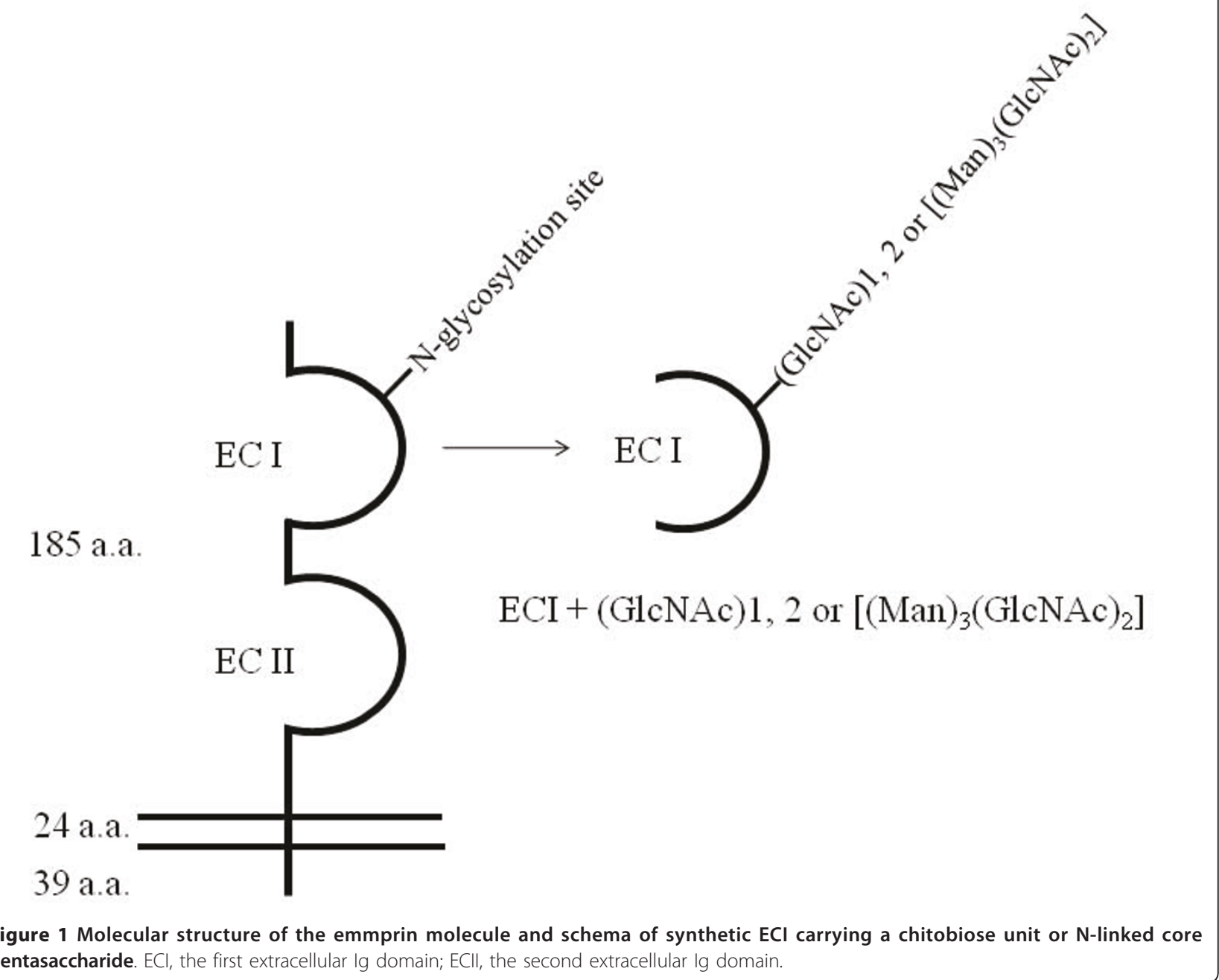


diameter wells containing $1.0 \mathrm{ml}$ DMEM with 10\% FBS. For co-cultures, the same number $\left(4 \times 10^{4}\right)$ of fibroblasts and tumor cells were incubated. The cells were allowed to attach for $24 \mathrm{~h}$ at $37^{\circ} \mathrm{C}$, after which their culture media were replaced with fresh serum-free (SF) DMEM containing $0.2 \%$ lactalbumin hydrolysate $(\mathrm{LH})$ $(0.5 \mathrm{ml} /$ well $)$ prior to the start of the experiment. Experiments under each condition were performed in triplicate wells. The culture media were replaced with fresh SF DMEM at 3 days and harvested at 6 days. The harvested media were used for immunoblotting and enzyme immunoassay.

Treatment of fibroblasts with $\mathrm{ECl}-(\mathrm{GlCNAC})_{1}$ or ${ }_{2}, \mathrm{ECl}-\left[(\mathrm{Man})_{3}\right.$ (GICNAC) 2 ], Asn44-(GICNAC) ${ }_{2}$, or ECl alone

Fibroblasts were allowed to attach for $24 \mathrm{~h}$ in DMEM with $10 \%$ FBS, then their culture media were replaced with fresh SF DMEM containing $0.2 \% \mathrm{LH}$ and ECI peptides with or without chitobiose units at various concentrations. Experiments under each condition were performed in triplicate wells. The culture media and cells were harvested at 3 days, and used for immunoblotting, enzyme immunoassay, RNA extraction and Northern blot analysis. For Northern blot analysis, cells in triplicate wells were combined to isolate poly (A)+RNA enough for the assay.

Inhibition experiments using anti-emmprin blocking antibody (UM-8D6, Ancell Corporation, Bayport, MN) were performed as described previously [23]. Briefly, ECI peptides with chitobiose units [ECI-(GlcNAc) $)_{2}$ ] were preincubated with the blocking antibody in SF DMEM containing $0.2 \% \mathrm{LH}$ at $37^{\circ} \mathrm{C}$ for $45 \mathrm{~min}$ and then added to fibroblasts $\left(4 \times 10^{4}\right)$. Their media were replaced once with fresh SF DMEM containing [ECI-(GlcNAc) $)_{2}$ ] and the blocking antibody at 3 days, and the culture media were harvested at 6 days.

\section{RNA isolation and Northern blot analysis}

Poly(A)+RNA was isolated from cultured cells using the Fast Track mRNA isolation kit (Invitrogen, San Diego, CA). Northern blotting was performed as described previously [9]. Cloned cDNA (1,752 bp) encoding human MMP-2 was used as a probe. For internal control of loading, the blots were subsequently hybridized to a glyceraldehyde-3-phosphate dehydrogenase (G3PDH) probe (Clontech, Mountain View, CA). The probes were radiolabeled by random priming with ${ }^{32} \mathrm{P}-\mathrm{CTP}$. For quantification of the RNA blot analysis, the radioactivity of mRNA signals for MMP-2 was directly measured by a Bioimaging Analyzer, FUJIX BAS2000 system (Fuji Photo Film, Tokyo, Japan), and normalized relative to the corresponding G3PDH mRNA signals.

\section{Immunoblotting}

SDS-PAGE and immunoblotting of conditioned media were performed using a 5-15\% gradient separating gel,
Immobilon membrane (Millipore, Bedford, MA) and a mouse mAb to human MMP-2 (75-7F7, Fuji Chemical Industries, Takaoka, Japan) as described previously [23]. To quantitate the relative amounts of the MMPs, the bands on the film were subjected to image analysis (Adobe Photoshop). Statistical analysis was performed using Student's $t$-test.

\section{Enzyme immunoassay (EIA)}

The protein concentrations of pro-MMP-2 were measured using commercially available EIA kit (Daiichi Fine Chemical, Takaoka, Japan) using the protocols supplied by the manufacturer. All assays were performed in triplicate and statistical analyses were performed using Student's $t$-test.

\section{Zymography}

Gelatinolytic activities in conditioned media were demonstrated using gelatin as a substrate. SDS-PAGE was performed under non-reducing conditions using a $9 \%$ separating gel containing $1 \mathrm{mg} / \mathrm{ml}$ gelatin. After electrophoresis, the gel was shaken gently in detergent buffer ( $5 \mathrm{mM} \mathrm{CaCl}_{2}, 2.5 \%$ Triton $\mathrm{X}-100$, and $50 \mathrm{mM}$ Tris- $\mathrm{HCl}$, $\mathrm{pH}$ 7.6) at room temperature for $60 \mathrm{~min}$ to remove the SDS, and then incubated in reaction buffer $(0.15 \mathrm{M} \mathrm{NaCl}$, $10 \mathrm{mM} \mathrm{CaCl}_{2}, 0.02 \% \mathrm{NaN}_{3}$, and $50 \mathrm{mM}$ Tris- $\mathrm{HCl}, \mathrm{pH} 7.6$ ) at $37^{\circ} \mathrm{C}$ for $30 \mathrm{~h}$ followed by staining with $2.5 \%$ Coomassie brilliant blue in $30 \%$ methanol and 10\% acetate. Enzyme activity was detected as a clear band on the resulting blue background of undigested gelatin. Gel images were analyzed digitally (Adobe Photoshop) to quantitate relative activities.

\section{Results}

Effects of ECl with or without a chitobiose unit on MMP production by fibroblasts

Previous studies showed two important findings with regard to emmprin activity, namely that the activity resides in ECI [6], and that it depends on N-glycosylation of extracellular domains of emmprin $[17,18]$. These findings prompted us to synthesize the whole ECI with attached sugar chains (N-glycosylation). In the first step, ECI carrying one or two GlcNAc at $\mathrm{Asn}^{44}$ was synthesized. When added to fibroblast cultures, ECI-(GlcNAc) ${ }_{1}$ or 2 stimulated MMP-2 mRNA expression, while unsubstituted ECI had no stimulatory effect (Figure 2). The stimulatory activity of ECI-(GlcNAc $)_{2}$ was much more than that of ECI(GlcNAc) $)_{1}$ and was dose-dependent: approximately 1.1-, 3.6 - and 4.6-fold stimulation at 7, 14 and $35 \mu \mathrm{M}$, respectively, compared to control. Stimulation was also demonstrated at the protein level. By immunoblotting, ECI-(GlcNAc) ${ }_{1}$ stimulated MMP-2 production by approximately 2.0-, 3.4- and 4.9-fold at 7, 14 and $35 \mu \mathrm{M}$, respectively, compared with the control, and ECI-(GlcNAc $)_{2}$ 


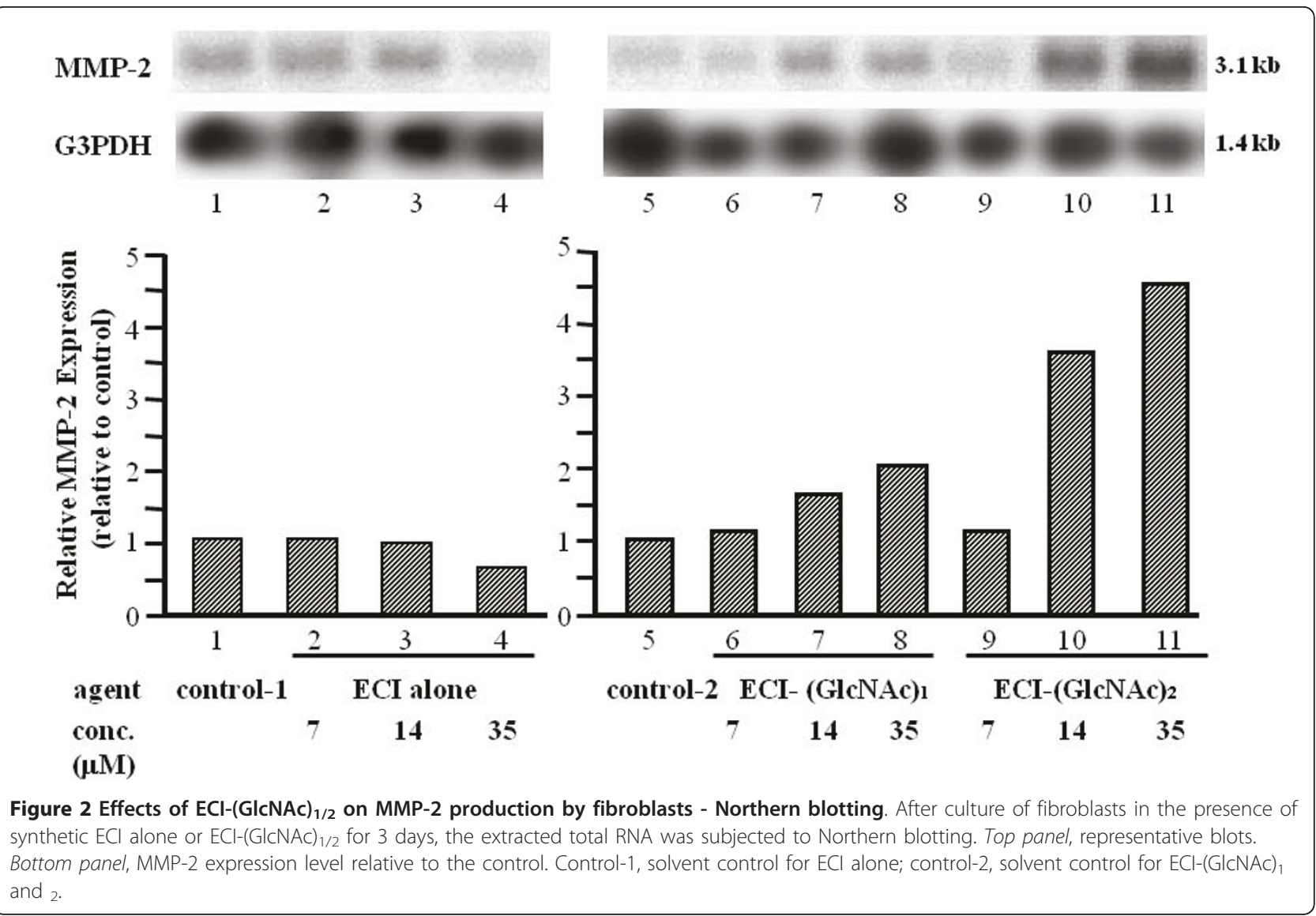

stimulated it by approximately 6.7-, 7.2- and 8.6-fold, respectively, at the same concentrations (Figure 3). Again, unsubstituted ECI had no stimulatory effect. The stimulated production of MMP-2 protein by ECI- $(\mathrm{GlcNAc})_{2}$ was also confirmed by quantitative EIA (Figure 4). ECI$(\text { GlcNAc })_{2}$ significantly stimulated MMP-2 production (mean \pm SEM, $114 \pm 9.8$ and $118 \pm 8.6 \mathrm{ng} / \mathrm{ml}$ ) of MMP-2 at 7 and $35 \mu \mathrm{M}$ respectively, compared to control $(9.1 \pm$ $2.4 \mathrm{ng} / \mathrm{ml})$ and ECI alone $(14.2 \pm 3.3$ and $15.2 \pm 4.1 \mathrm{ng} / \mathrm{ml}$ at 7 and $35 \mu \mathrm{M}$, respectively) $(p<0.01)$. The levels of MMP-2 stimulated with ECI-(GlcNAc) $)_{2}$ at 7 or $35 \mu \mathrm{M}$ were similar to those stimulated in tumor cell-fibroblast co-cultures $(124 \pm 14 \mathrm{ng} / \mathrm{ml})$.

To rule out that (GlcNAc) $)_{2}$ alone stimulated MMP synthesis in fibroblasts, Asn-(GlcNAc) ${ }_{2}$ was added to fibroblast cultures. The results showed no stimulatory effect for Asn-(GlcNAc) $)_{2}$ or unsubstituted ECI on MMP-2 production (Figure 5), while ECI-(GlcNAc) ${ }_{2}$ stimulated MMP-2 production.

MMP-2 stimulation activity of ECl-(GlcNAc) $)_{2}$ vs. ECI-[(Man $)_{3}$ $\left.(\mathrm{GlcNAc})_{2}\right]$

Next, we investigated whether ECI with longer chitobiose units could induce more potent stimulation of fibroblasts to produce larger amounts of MMP-2. Treatment with ECI-[(Man $\left.)_{3}(\text { GlcNAc })_{2}\right]$ significantly stimulated fibroblasts to produce MMP-2 $(98.7 \pm 5.4$ and $106 \pm 11 \mathrm{ng} / \mathrm{ml}$ at 7 and $35 \mu \mathrm{M}$, respectively) compared to the control (24.4 \pm $3.2 \mathrm{ng} / \mathrm{m})(p<0.01)$ (Figure 6A). However, the extents of stimulation by ECI- $\left[(\mathrm{Man})_{3}(\mathrm{GlcNAc})_{2}\right]$ were lower than those by the same dose of ECI-(GlcNAc) ${ }_{2}(132 \pm 7.8$ and $138 \pm 8.5$ at 7 and $35 \mu \mathrm{M}$, respectively). The extents of stimulation of MMP-2 production by ECI-(GlcNAc) $)_{2}$ were almost similar to those by FU-EPS-1 tumor cell surface emmprin in co-cultures of FU-EPS-1 cells and fibroblasts (Figure 6B).

\section{Anti-emmprin blocking antibody Inhibits the MMP-2 stimulating activity of $\mathrm{ECl}-(\mathrm{GlCNAC})_{2}$ peptide}

To confirm that the responses in the above ECI(GlcNAc) $)_{2}$ peptide-induced stimulation of MMP-2 production were emmprin-mediated, inhibition assay using anti-emmprin blocking antibody was performed. EIA showed that the anti-emmprin blocking antibody inhibited $(14 \mu \mathrm{M})$ ECI-(GlcNAc) $)_{2}$-induced stimulation of MMP-2 production (inhibition by approximately $58 \%$ at $10 \mu \mathrm{g} / \mathrm{ml}$ and $54 \%$ at $50 \mu \mathrm{g} / \mathrm{ml}$ ), while non-immune IgG as control did not cause any inhibition (Figure 7). 


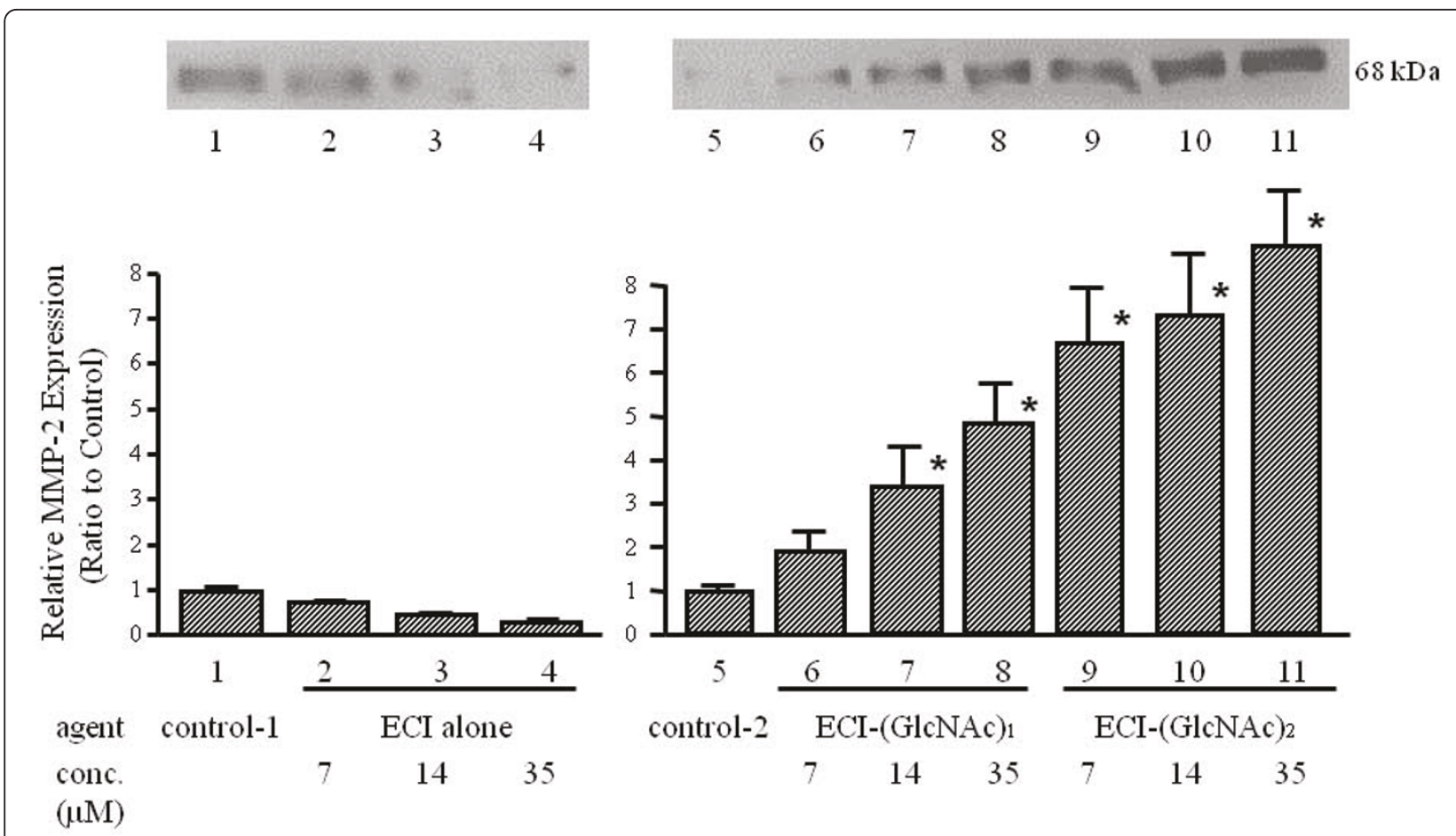

Figure 3 Effects of ECI-(GICNAc) $)_{1 / 2}$ on MMP-2 production by fibroblasts - western blotting. After culture of fibroblasts in the presence of synthetic $\mathrm{ECl}$ alone or $\mathrm{ECl}-(\mathrm{GlCNAc})_{1 / 2}$ for 3 days, the conditioned media were subjected to western blotting. Top panel, representative blots. Bottom panel, MMP-2 expression level relative to the control. Data are mean \pm SEM $(n=3)$. Control-1, solvent control for $\mathrm{ECl}$ alone; control-2, solvent control for $\mathrm{ECl}-(\mathrm{GlCNAc})_{1}$ and $2 .{ }^{*} p<0.01$, compared with the control.

\section{Discussion}

The present study showed that the synthetic emmprin ECI peptide can exhibit emmprin activity to stimulate fibroblasts to produce MMP-2, when substituted with a chitobiose unit (GlcNAc-GlcNAc). The whole ECI without a chitobiose unit showed no stimulatory activity on MMP-2 production. These findings suggest that ECI is a key domain in determining the activity of the emmprin molecule. Moreover, the results showed that ECI was active, in terms of stimulating MMP-2 production, in the presence of the shortest form of $\mathrm{N}$-glycosylation.

Two groups have reported that emmprin stimulation of MMP production in fibroblasts is dependent on N-glycosylation of its extracellular domains $[17,18]$. The ability of the emmprin molecule to participate in homophilic adhesion alone is not sufficient for MMP induction because glycosylation is not needed for homophilic adhesion but is essential for MMP-1 and MMP-2 induction [18]. ECI with glycosylation, although just a short chitobiose unit, may send a signal into the cells to upregulate MMP-2. There are two possibilities: First, the carbohydrate side chains may participate directly in ligand binding to a putative fibroblast receptor. Second, they may be necessary for preservation of an active molecular conformation [5]. Intriguingly, in our study, ECI substituted with only two GlcNAcs induced MMP-2 expression at both mRNA and protein levels. This chitobiose unit is the common structure formed at the reducing end of $\mathrm{N}$-linked carbohydrates. Longer and more complex $\mathrm{N}$-linked carbohydrates are reported to be involved in cellular adhesion, possibly via direct binding to lectins [26]. As for emmprin ECI, however, substitution with only two GlcNAcs was more potent than that with the core pentasaccharide. Thus, it is more likely that the chitobiose unit plays a role in preservation of an active molecular conformation. The peptide structures might be bent somehow by hydrogen bonds formed between amino acids and carbohydrates to form an active molecular structure. This possibility is currently under investigation in our laboratories. Similarly, it was shown that the adhesion domain of human CD2 bears a single Nlinked carbohydrate, and that the carbohydrate stabilizes the protein fold by counterbalancing the unfavorable clustering of five positive charges centered about lysine-61 of CD2 [27]. Furthermore, the addition of short saccharides (e.g., di-, tri- and tetra-saccharides) to Notch by Fringe is postulated to increase Notch's sensitivity to Delta-like ligands, although they are O-linked carbohydrates [28]. This glycosylation supposedly does not affect Notch signaling by directly influencing the binding between Notch and ligands but may influence steps that occur just before the 


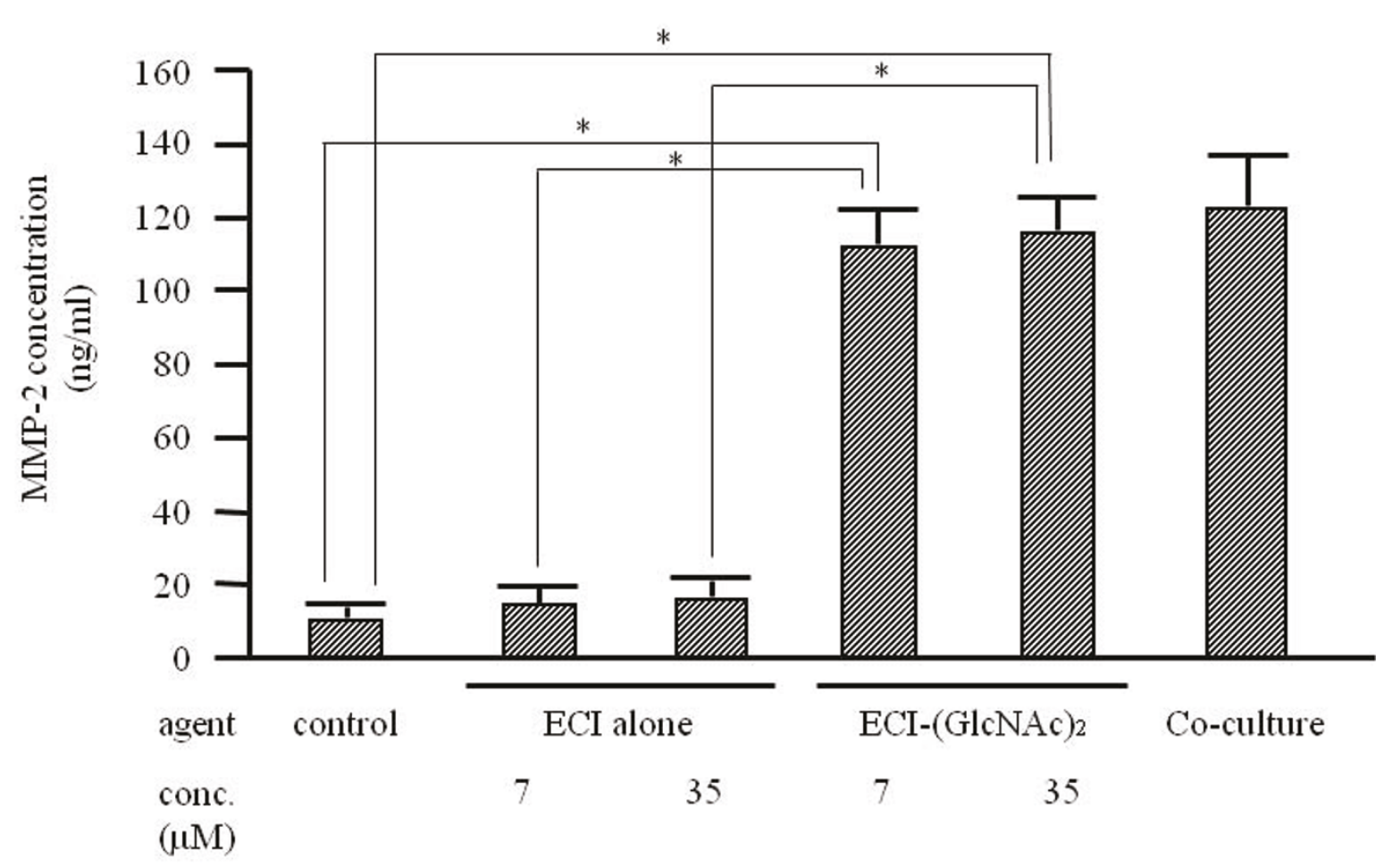

Figure 4 Effects of ECl-(GICNAc) $1 / 2$ on MMP-2 production by fibroblasts - enzyme immunoassay. After culture of fibroblasts in the presence of synthetic $\mathrm{ECl}$ alone or $\mathrm{ECl}-(\mathrm{GlcNAC})_{1 / 2}$ for 3 days, the conditioned media were subjected to enzyme immunoassay. Data are mean \pm SEM $(n=3)$. Control, solvent control for $\mathrm{ECl}$ alone and $\mathrm{ECl}-(\mathrm{GlCNAC})_{2} .{ }^{*} p<0.01$, compared with the control.

release of the Notch intracellular domain by proteolytic cleavages [28].

Induction of MMP-1, 2 and 3 mRNAs in uterine fibroblasts by non-glycosylated recombinant emmprin has been reported also [19], although a higher expression at the protein level was not confirmed. The authors hypothesized that their recombinant emmprin maintained the disulfide-stabilized tertiary structures, which let emmprin form dimers in solution and promoted their binding to the cell surface receptors, followed by increased MMP expression. Our study used smaller ECI peptides so that chitobiose substitution might have been necessary to give the peptide some tertiary structures.
Moreover, the fibroblasts used in this study were stimulated to produce only MMP-2, but not MMP-1 and 3, in response to tumor cell emmprin (data not shown). Thus, the effect of the ECI peptide with chitobiose substitution on MMP-1 and 3 could not be examined.

Recently, the crystal structure of the extracellular portion of emmprin has been determined [29]. The structure comprises an $\mathrm{N}$-terminal IgC2 domain (corresponding to $\mathrm{ECI}$ in this paper) and a C-terminal IgI domain, which are connected by a 5 -residue flexible linker. This unique domain organization leads to overall flexibility and diverse dimerization manners of emmprin and may explain its multifunctional character. Another possible explanation

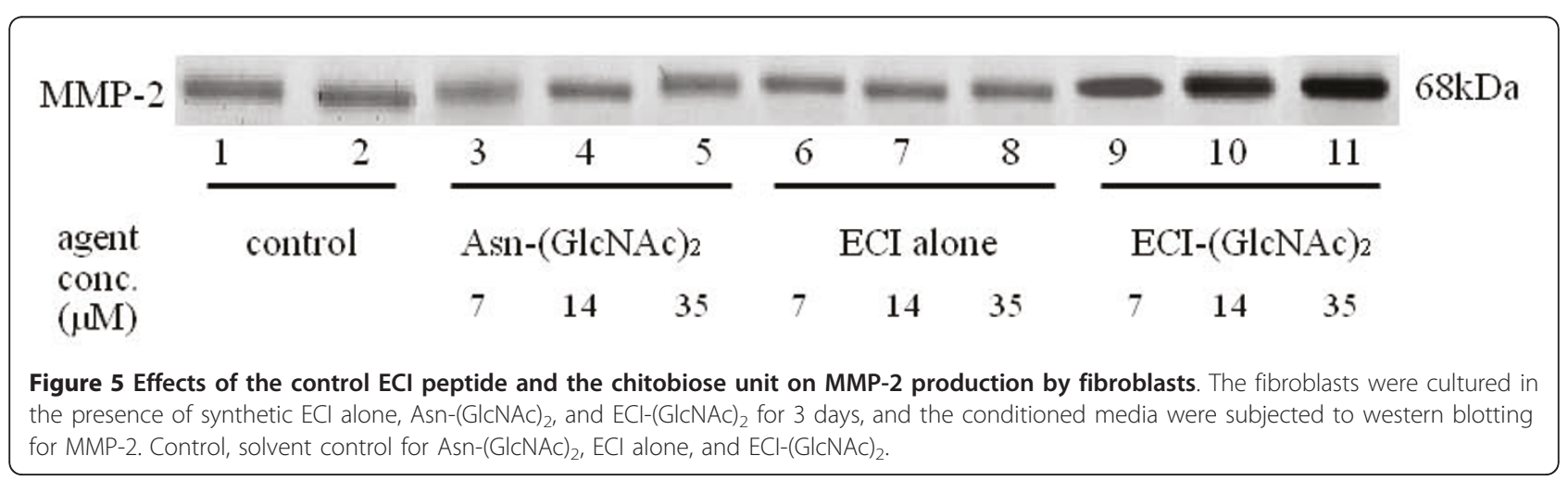




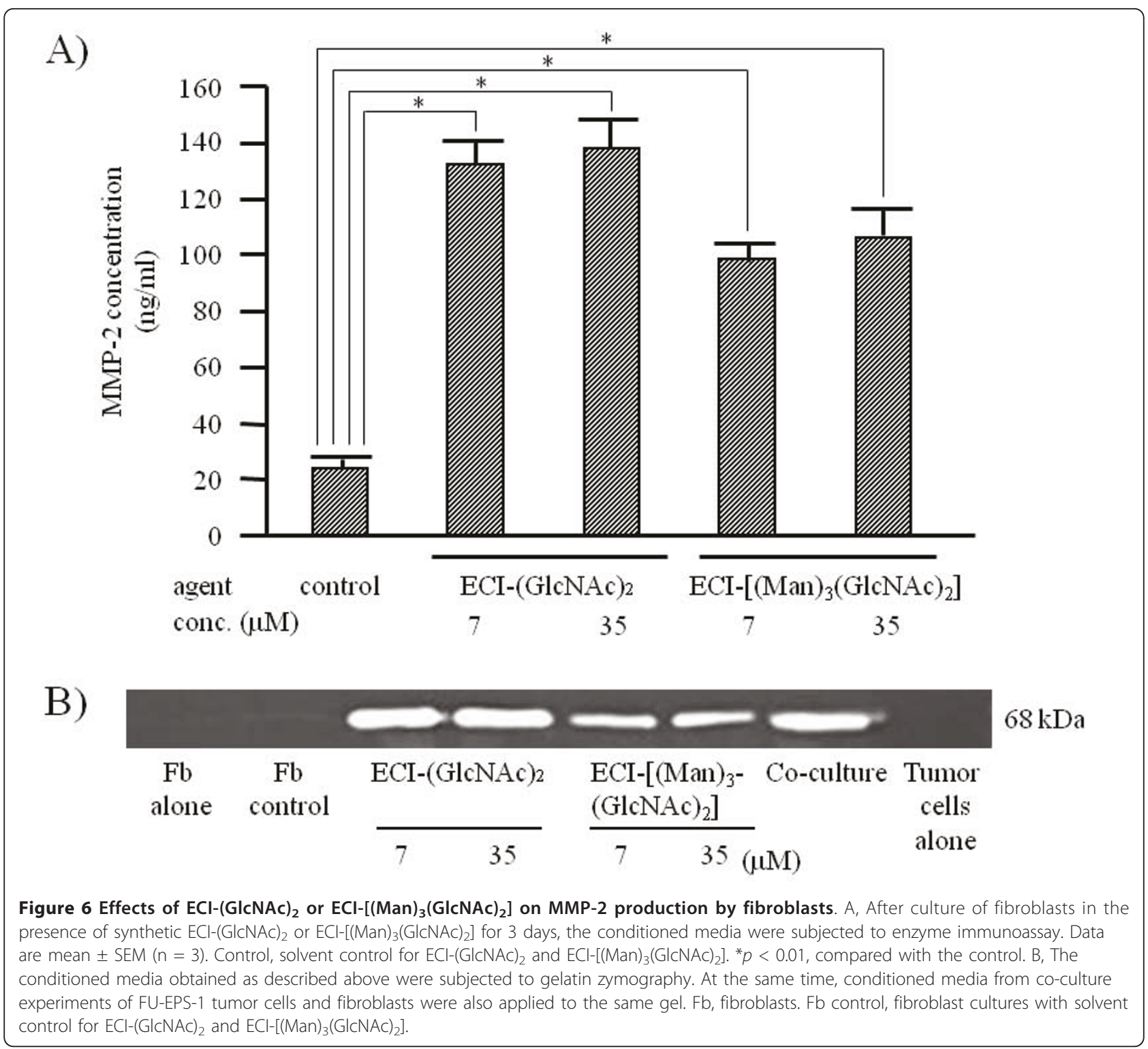

for multifunction is glycosylation, since the latter is a highly diverse nontemplate-driven process that can generate enormous informational content [30]. The glycosylated form of emmprin was not analyzed in the above mentioned crystalographic study.

In this study, ECI was shown to stimulate MMP-2 production in the presence of the shortest form of $\mathrm{N}$ glycosylation. On the contrary, we previously reported that ECI-derived peptides inhibit emmprin-enhanced MMP-2 production in the absence of $\mathrm{N}$-glycosylation $[11,24,31]$. One of the ECI-derived peptides, emp\#2, which contains a putative $\mathrm{N}$-glycosylation site but shows no glycosylation, effectively inhibited emmprin-induced stimulation of MMP-2 production. Furthermore, emp\#2 peptide effectively inhibited the enhanced invasion of matrigel by glioblastoma cells in the co-presence of fibroblasts [31]. These results also show biological importance of presence of $\mathrm{N}$-glycosylation in ECI.

Thus far, several lines of evidence point to the importance of emmprin in cancer biology. First, emmprin is upregulated in a broad spectrum of malignant tumors, including carcinomas, gliomas and lymphomas, compared with their normal counterparts as mentioned in the Introduction section. Second, emmprin promotes tumor progression as well as upregulation of MMPs in animal models: emmprin cDNA-transfected human breast cancer cells were considerably more tumorigenic and invasive than plasmid-transfected cancer cells when implanted into the nude mouse [32]. Third, as already discussed above, emmprin may be responsible for upregulated MMPs, 


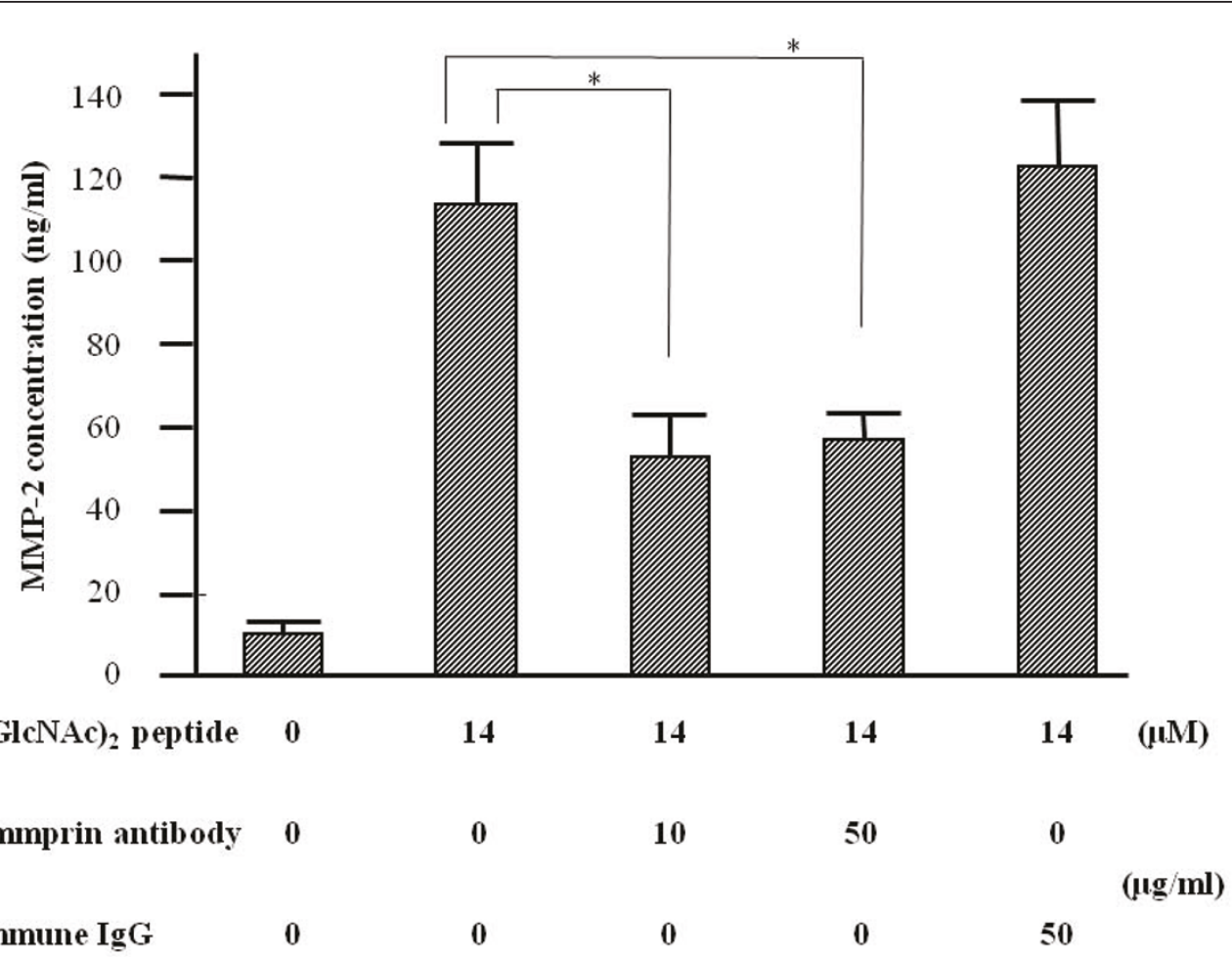

Figure 7 Effects of anti-emmprin antibody on ECI-(GICNAC) ${ }_{2}$-induced stimulation of MMP-2 production by fibroblasts. Fibroblasts were cultured in the presence of $\mathrm{ECl}-(\mathrm{GlCNAC})_{2}$ and anti-emmprin antibody or control non-immune lgG for 3 days, and the conditioned media were subjected to enzyme immunoassay for MMP-2. Data are mean \pm SEM $(n=4) .{ }^{*} p<0.01$, compared with the control.

enhanced angiogenesis and hyaluronic acid production, transformation of stroma fibroblasts, and drug resistance $[6,12-18,32]$. In this context, it is important to elucidate the mechanisms involved in multifunctionality of the emmprin molecule.

\section{Conclusions}

This study indicates that ECI can mimic emmprin activity in terms of stimulating MMP-2 production by fibroblasts when substituted with chitobiose, the disaccharide with which $\mathrm{N}$-glycosylation starts. This synthetic glycopeptide may be useful for elucidating the mechanisms involved in glycosylation-mediated emmprin activities, which could lead to new strategies to prevent cancer invasion and progression enhanced by emmprin.

\section{Acknowledgements}

The authors thank Ms. M. Onitsuka for the skillful assistance in cell culture and co-culture experiments. This work was supported in part by a Grant-inAid for Scientific Research (No. 20590415) from the Ministry of Education, Culture, Sports, Science, and Technology of Japan, a grant from the Research Center for Advanced Molecular Medicine, Fukuoka University, and NIH grant CA082867.

\section{Author details}

'Department of Pathology, Fukuoka University School of Medicine, 7-45-1 Nanakuma, Jonan-ku, Fukuoka 814-0180, Japan. ${ }^{2}$ Department of Thoracic
Surgery, Fukuoka University School of Medicine, 7-45-1 Nanakuma, Jonan-ku, Fukuoka 814-0180, Japan. ${ }^{3}$ Department of Neurosurgery, NTT Medical Center Tokyo, 5-9-22 Higashigotanda, Shinagawa-ku, Tokyo 141-8625, Japan. ${ }^{4}$ Department of Applied Biochemistry, Institute of Glycotechnology, 1117 Kitakinme, Hiratsuka, Tokai University, Kanagawa 259-1292, Japan. ${ }^{5}$ Department of Regenerative Medicine and Cell Biology, Medical University of South Carolina, 173 Ashley Avenue, Charleston, SC 29425, USA. ${ }^{6}$ Shimane University Hospital Cancer Center, 89-1 Enya, Izumo, Shimane 693-8501 Japan. ${ }^{7}$ Department of Pathology, Keio University School of Medicine, 35 Shinanomachi, Tokyo 160-0016, Japan.

\section{Authors' contributions}

TK, TS, HH and KK carried out the experiments. KN, HH, BPT, JS and Al participated in the design of the study, its coordination, and helped to draft the manuscript. In addition, technical guidance from YN and YO lead to successful experiments. All authors approved the final manuscript.

\section{Competing interests}

The authors declare that they have no competing interests.

Received: 21 February 2011 Accepted: 17 July 2011

Published: 17 July 2011

\section{References}

1. Stetler-Stevenson WG, Aznavoorian S, Liotta LA: Tumor cell interactions with the extracellular matrix during invasion and metastasis. Annu Rev Cell Biol 1993, 9:541-573.

2. Poulsom R, Pignatelli M, Stetler-Stevenson WG, Liotta LA, Wright PA, Jeffery RE, Longcroft JM, Rogers L, Stamp GW: Stromal expression of 72 kda type IV collagenase (MMP-2) and TIMP-2 mRNAs in colorectal neoplasia. Am J Pathol 1992, 141(2):389-396.

3. Poulsom R, Hanby AM, Pignatelli M, Jeffery RE, Longcroft JM, Rogers L, Stamp GW: Expression of gelatinase A and TIMP-2 mRNAs in 
desmoplastic fibroblasts in both mammary carcinomas and basal cell carcinomas of the skin. J Clin Pathol 1993, 46(5):429-436.

4. Pyke C, Ralfkiaer E, Tryggvason K, Dano K: Messenger RNA for two type IV collagenases is located in stromal cells in human colon cancer. Am J Pathol 1993, 142(2):359-365.

5. Toole BP: Emmprin (CD147), a cell surface regulator of matrix metalloproteinase production and function. Curr Top Dev Biol 2003, 54:371-389.

6. Biswas C, Zhang Y, DeCastro R, Guo H, Nakamura T, Kataoka H, Nabeshima K: The human tumor cell-derived collagenase stimulatory factor (renamed EMMPRIN) is a member of the immunoglobulin superfamily. Cancer Res 1995, 55(2):434-439.

7. Muraoka K, Nabeshima K, Murayama T, Biswas C, Koono M: Enhanced expression of a tumor-cell-derived collagenase-stimulatory factor in urothelial carcinoma: its usefulness as a tumor marker for bladder cancers. Int J Cancer 1993, 55(1):19-26.

8. Polette M, Gilles C, Marchand V, Lorenzato M, Toole B, Tournier JM, Zucker S, Birembaut P: Tumor collagenase stimulatory factor (TCSF) expression and localization in human lung and breast cancers. J Histochem Cytochem 1997, 45(5):703-709.

9. Sameshima T, Nabeshima K, Toole BP, Yokogami K, Okada Y, Goya T, Koono M, Wakisaka S: Expression of emmprin (CD147), a cell surface inducer of matrix metalloproteinases, in normal human brain and gliomas. Int J Cancer 2000, 88(1):21-27.

10. Kanekura T, Chen X, Kanzaki T: Basigin (CD147) is expressed on melanoma cells and induces tumor cell invasion by stimulating production of matrix metalloproteinases by fibroblasts. Int I Cancer 2002, 99(4):520-528

11. Nabeshima K, Suzumiya J, Nagano M, Ohshima K, Toole BP, Tamura K, Iwasaki H, Kikuchi M: Expression of emmprin, a cell surface inducer of matrix metalloproteinases (MMP), in T-cell lymphomas. J Pathol 2004, 202(3):341-351.

12. Tang $Y$, Nakada MT, Kesavan $P$, McCabe F, Millar H, Rafferty $P$, Bugelski $P$, Yan L: Extracellular matrix metalloproteinase inducer stimulates tumor angiogenesis by elevating vascular endothelial cell growth factor and matrix metalloproteinases. Cancer Res 2005, 65(8):3193-3199.

13. Huet E, Vallee B, Szul D, Verrecchia F, Mourah S, Jester JV, Hoang-Xuan T, Menashi S, Gabison EE: Extracellular matrix metalloproteinase inducer/ CD147 promotes myofibroblast differentiation by inducing alphasmooth muscle actin expression and collagen gel contraction: implications in tissue remodeling. Faseb J 2008, 22(4):1144-1154

14. Misra S, Ghatak S, Zoltan-Jones A, Toole BP: Regulation of multidrug resistance in cancer cells by hyaluronan. J Biol Chem 2003, 278(28):25285-25288

15. Yang JM, Xu Z, Wu H, Zhu H, Wu X, Hait WN: Overexpression of extracellular matrix metalloproteinase inducer in multidrug resistant cancer cells. Mol Cancer Res 2003, 1(6):420-427.

16. Marieb EA, Zoltan-Jones A, Li R, Misra S, Ghatak S, Cao J, Zucker S, Toole BP. Emmprin promotes anchorage-independent growth in human mammary carcinoma cells by stimulating hyaluronan production. Cancer Res 2004, 64(4):1229-1232.

17. Guo H, Zucker S, Gordon MK, Toole BP, Biswas C: Stimulation of matrix metalloproteinase production by recombinant extracellular matrix metalloproteinase inducer from transfected Chinese hamster ovary cells. J Biol Chem 1997, 272(1):24-27.

18. Sun J, Hemler ME: Regulation of MMP-1 and MMP-2 production through CD147/extracellular matrix metalloproteinase inducer interactions. Cancer Res 2001, 61(5):2276-2281.

19. Belton RJ, Chen L, Mesquita FS, Nowak RA: Basigin-2 is a cell surface receptor for soluble basigin ligand. $J$ Biol Chem 2008, 283(26):17805-17814.

20. Aimoto S: Polypeptide synthesis by the thioester method. Biopolymers 1999, 51(4):247-265.

21. Hojo H, Watabe J, Nakahara Y, Nakahara Y, Ito Y, Nabeshima K, Toole BP: Synthesis of the extracellular Ig domain I of emmprin carrying a chitobiose unit. Tetrahedron Lett 2001, 42:3001-3004

22. Hojo H, Haginoya E, Matsumoto $Y$, Nakahara $Y$, Nabeshima $K$, Toole BP, Watanabe $\mathrm{Y}$ : The first synthesis of peptide thioester carrying $\mathrm{N}$-linked core pentasaccharide through modified Fmoc thioester preparation: synthesis of an N-glycosylated Ig domain of emmprin. Tetrahedron Lett 2003, 44:2961-2964.
23. Sameshima T, Nabeshima K, Toole BP, Yokogami K, Okada Y, Goya T, Koono M, Wakisaka S: Glioma cell extracellular matrix metalloproteinase inducer (EMMPRIN) (CD147) stimulates production of membrane-type matrix metalloproteinases and activated gelatinase $\mathrm{A}$ in co-cultures with brain-derived fibroblasts. Cancer Lett 2000, 157(2):177-184.

24. Koga K, Nabeshima K, Aoki M, Kawakami T, Hamasaki M, Toole BP, Nakayama J, Iwasaki H: Emmprin in epithelioid sarcoma: expression in tumor cell membrane and stimulation of MMP-2 production in tumorassociated fibroblasts. Int I Cancer 2007, 120(4):761-768.

25. Nishio J, Iwasaki H, Nabeshima K, Ishiguro M, Naumann S, Isayama T, Naito M, Kaneko Y, Kikuchi M, Bridge JA: Establishment of a new human epithelioid sarcoma cell line, FU-EPS-1: molecular cytogenetic characterization by use of spectral karyotyping and comparative genomic hybridization. Int J Oncol 2005, 27(2):361-369.

26. Akama TO, Nakagawa H, Sugihara K, Narisawa S, Ohyama C, Nishimura S, O'Brien DA, Moremen KW, Millan JL, Fukuda MN: Germ cell survival through carbohydrate-mediated interaction with Sertoli cells. Science 2002, 295(5552):124-127.

27. Berditchevski F, Chang S, Bodorova J, Hemler ME: Generation of monoclonal antibodies to integrin-associated proteins. Evidence that alpha3beta1 complexes with EMMPRIN/basigin/OX47/M6. J Biol Chem 1997, 272:29174-29180

28. Moloney DJ, Panin VM, Johnston SH, Chen J, Shao L, Wilson R, Wang Y, Stanley $P$, Irvine KD, Haltiwanger RS, et al: Fringe is a glycosyltransferase that modifies Notch. Nature 2000, 406(6794):369-375.

29. Yu XL, Hu T, Du JM, Ding JP, Yang XM, Zhang J, Yang B, Shen X, Zhang Z, Zhong WD, et al: Crystal structure of HAb18G/CD147: implications for immunoglobulin superfamily homophilic adhesion. J Biol Chem 2008, 283(26):18056-18065

30. Trzpis M, McLaughlin PM, de Leij LM, Harmsen MC: Epithelial cell adhesion molecule: more than a carcinoma marker and adhesion molecule. Am J Pathol 2007, 171(2):386-395.

31. Koga K, Aoki M, Sameshima T, Hamasaki M, Egawa N, Seiki M, Toole BP, Suzumiya J, Nabeshima K: Synthetic emmprin peptides inhibit tumor cellfibroblast interaction-stimulated upregulation of MMP-2 and tumor cell invasion. Int J Oncol 2011, 39(3):657-664.

32. Zucker S, Hymowitz M, Rollo EE, Mann R, Conner CE, Cao J, Foda HD, Tompkins DC, Toole BP: Tumorigenic potential of extracellular matrix metalloproteinase inducer. Am J Pathol 2001, 158(6):1921-1928.

\section{Pre-publication history}

The pre-publication history for this paper can be accessed here: http://www.biomedcentral.com/1471-2407/11/300/prepub

\section{doi:10.1186/1471-2407-11-300}

Cite this article as: Kawakami et al: Synthetic emmprin peptides with chitobiose substitution stimulate MMP-2 production by fibroblasts. BMC Cancer 2011 11:300.

\section{Submit your next manuscript to BioMed Central and take full advantage of:}

- Convenient online submission

- Thorough peer review

- No space constraints or color figure charges

- Immediate publication on acceptance

- Inclusion in PubMed, CAS, Scopus and Google Scholar

- Research which is freely available for redistribution 